Revue d'histoire de l'Amérique française

REVUE D.HISTOIRE DE L'AMÉRIQUE FRANÇAISE

\title{
Les attitudes devant la mort : sur deux ouvrages récents
}

\section{Guy Laperrière}

Volume 32, numéro 2, septembre 1978

URI : https://id.erudit.org/iderudit/303693ar

DOI : https://doi.org/10.7202/303693ar

Aller au sommaire du numéro

Éditeur(s)

Institut d'histoire de l'Amérique française

ISSN

0035-2357 (imprimé)

1492-1383 (numérique)

Découvrir la revue

Citer cette note

Laperrière, G. (1978). Les attitudes devant la mort : sur deux ouvrages récents. Revue d'histoire de l'Amérique française, 32(2), 251-255.

https://doi.org/10.7202/303693ar d'utilisation que vous pouvez consulter en ligne.

https://apropos.erudit.org/fr/usagers/politique-dutilisation/ 


\title{
LES ATTITUDES DEVANT LA MORT: SUR DEUX OUVRAGES RÉCENTS*
}

\author{
GUY LAPPERIÈRE \\ Département d'bistoire \\ Université de Sherbrooke
}

Deux ouvrages parus au même moment viennent solliciter notre attention sur le phénomène de la mort. Loin de moi de mettre sur le même pied la grande œuvre de Philippe Ariès, L'homme devant la mort et le petit volume de Paul Jacob, Les revenants de la Beauce. Mais bien que ni l'un ni l'autre ne touchent directement l'histoire de l'Amérique française, ils appellent, chacun à sa manière, à des recherches sur l'histoire de la mort au Québec.

On ne reviendra pas ici sur l'omniprésence qu'ont prise les études sur la mort dans l'histoire des mentalités depuis quelques années. Philippe Ariès, déjà connu pour ses études pionnières sur les populations et la famille d'Ancien Régime, a donc publié une synthèse qui s'ajoute aux travaux remarquables des Vovelle et Lebrun, mais qui a une ambition beaucoup plus grande, puisqu'elle voudrait recouvrir tout l'espace occidental sur une période de plus de mille ans! L'intérêt des historiens pour la mort n'est pas nouveau: dans l'après-guerre déjà, J. Huizinga, E. Morin, A. Tenenti, avaient publié des œuvres déterminantes pour l'histoire des idées. En réassumant ces études et en plongeant dans de nouvelles séries de documents, Ariès parvient à nous communiquer une première idéeforce, toujours nouvelle pour l'historien même si elle devrait bien être, à la longue, naturelle: la perception de la mort, les attitudes devant la mort, n'ont pas toujours été ce que nous croyions qu'elles

* Philippe Ariès, L'homme devant la mort (Paris, Seuil, 1977), 642 p. Paul Jacob, Les revenants de la Beauce (Montréal, Boréal express, 1977), 159 p. 
étaient. Des changements considérables se sont produits qu'Ariès aime appeler des «révolutions du sentiment». Là où l'on croyait trouver une image «traditionnelle» de la mort, remplacée au milieu du $\mathrm{XX}^{\mathrm{e}}$ siècle par une vision «nouvelle», on voit défiler, sur un millénaire, une série d'attitudes complètement différentes et qui, pour certains de leurs aspects, renouent les unes avec les autres pardessus les siècles. Voyons rapidement les principaux modèles que présente Ariès.

Le premier type retenu est celui du Haut Moyen Âge. On peut regretter que l'A. n'ait pas inclus dans sa synthèse les riches études sur les rites et l'art funéraires antiques, mais cela l'aurait pratiquement amené à doubler l'étendue d'un travail déjà considérable. Le premier Moyen Âge fait apparaître une proximité, une familiarité avec la mort qu'Ariès nomme joliment «la mort apprivoisée». C'est la mort de Roland, de Tristan, qu'on sent venir et qu'on annonce. Le mourant est entouré de ses proches, la mort est un sommeil, un repos, les cimetières sont situés au cœur des localités, ce sont même des lieux habités. À partir du XII ${ }^{\mathrm{e}}$ siècle, un deuxième modèle apparaît, où la mort devient une affaire individuelle, axée sur la préoccupation du salut personnel: c'est «la mort de soi», qui culminera dans les thèmes macabres du $\mathrm{XV}^{\mathrm{e}}$ siècle, symbole non pas tant d'une peur de la mort que d'un amour passionné de la vie. L'épitaphe, le monument commémoratif, le testament, sont les symboles de cette individualisation de la mort, à laquelle la théologie associera les messes pour les défunts et les confréries de pénitents. Du XVI au XVIII ${ }^{e}$ siècle, on assiste à un changement de la sensibilité qui nous fait passer de plus en plus de la mort «apprivoisée» à «la mort ensauvagée», qui restera le modèle dominant jusqu'au $\mathrm{XX}^{\mathrm{e}}$ siècle. C'est moins le moment de la mort que la fragilité de la vie qui devient le centre des préoccupations, ce qui fait perdre à la mort une grande part de son intensité. On retrouve donc beaucoup plus de simplicité dans la mort, on revient aux cimetières en plein air. Au $\mathrm{XVIII}^{\mathrm{e}}$ siècle apparaît la crainte d'être enterré vivant, première manifestation de la «Grande Peur de la Mort». C'est l'aboutissement d'une profonde transformation, où l'imaginaire force les défenses contre la nature longuement mises en place par la société et ce, tant du côté de la mort que du côté de l'amour: Éros et Thanatos deviennent beaucoup plus proches des hommes et sont souvent associés l'un à l'autre, comme on le voit dans les phénomènes de nécrophilie. C'est dans ce climat qu'apparait la mort romantique, qu'Ariès décrit longuement, non sans quelque complaisance. C'est «le temps 
des belles morts", où la douleur s'exprime à la mort de l'autre, surtout du proche parent; c'est l'époque où se multiplient les visites au cimetière, perçu comme un lieu sacré. La mort romantique, c'est le modèle de «la mort de toi», et l'on voit bien qu'il s'est maintenu jusqu'à nos jours. Un dernier modèle l'a cependant emporté au $\mathrm{XX}^{\mathrm{e}}$ siècle, celui de «la mort inversée» ou de «la mort interdite». Le mourant devient étranger à sa propre mort, on cache la mort, notamment à l'hôpital, on pratique l'incinération, on supprime le deuil. Et en même temps se devine une tendance à revenir à une mort à nouveau apprivoisée...

Ce qu'il y a d'intéressant dans l'ouvrage d'Ariès - et ce qui a déjà commencé à être controversé - , c'est le choix des «indicateurs» de mentalités. Ce sont principalement les tombeaux et les cimetières, les rites funéraires, les testaments, un certain nombre de traités savants, des œuvres littéraires, des œuvres d'art, ainsi que, pour la période contemporaine, des études de sociologues ou de psychologues. M. Vovelle s'est étonné de ne retrouver ici aucune analyse des traités de théologie ou des livres de piété, ni surtout d'étude statistique de la mortalité, qu'on aurait pu attendre d'un des pionniers de la démographie historique. Il n'est pas dans notre intention d'entrer dans ce débat méthodologique, mais plutôt d'indiquer quelques avenues que ce volume ouvre à la recherche historique québécoise. Signalons d'abord au lecteur pressé qu'il retrouvera tout l'essentiel de L'homme devant la mort dans les Essais sur l'histoire de la mort en Occident du Moyen Âge à nos jours, publiés deux ans plus tôt (Paris, Seuil, 1975, 225 p.). Notons ensuite qu'Ariès ne couvre pas ici tout le territoire occidental, tant s'en faut. En fait, c'est la France qui sert de pivot, avec de sérieuses incursions dans le monde anglosaxon (Grande-Bretagne et États-Unis) et quelques allusions à l'Allemagne, l'Espagne ou l'Italie. C'est assez cependant pour suggérer ce que l'expérience québécoise peut avoir d'original. Sur une période qui s'étend du XVII ${ }^{e}$ au $\mathrm{XX}^{\mathrm{e}}$ siècle, plusieurs types de sources utilisées par Ariès s'offrent au chercheur d'ici et permettraient de préciser, voire de nuancer, les modèles les plus récents présentés par Ariès, et peut-être même des plus anciens, si tant est que le Canada français a perpétué un certain nombre de traditions médiévales. Voyons quelques questions possibles parmi tant d'autres. Comment l'instant de la mort a-t-il été vécu? Quelle importance la famille, l'Église avaient-elles lors de l'agonie? Quels étaient les rites qui suivaient le décès? Comment a-t-on disposé, au fil des siècles, des corps des défunts? Quand a-t-on cessé d'exposer les morts dans les 
maisons? On pourrait écrire une histoire de l'apparition des salons funéraires, ou de l'industrie de ceux qu'on dénomme sans sourire les «entrepreneurs de pompes funèbres»! Une histoire des cimetières ne manquerait pas non plus d'intérêt. Comment expliquer la crainte que semble avoir inspiré aux patriotes de 1837, par exemple, la menace de n'être pas inhumés «en terre sainte»? Les cimetières ont-ils toujours appartenu aux Églises, ou, comme on peut le soupçonner en rencontrant de petits cimetières abandonnés au détour de si nombreuses routes des Cantons de l'Est, le cimetière a-t-il été à une certaine époque, privé, voire familial? Comment enterrait-on au moment d'épidémies? Depuis quand et pourquoi recherche-t-on avec tant d'acharnement les corps disparus des incendiés ou des noyés? Comment les morts ont-ils survécu dans la mémoire collective? A-t-on toujors prié pour les morts? Le fait-on surtout en faisant dire des messes? Y a-t-il des confréries qui se sont occupées des morts, des âmes du purgatoire? En somme, comment ont évolué au Québec les pratiques, les attitudes, les croyances concernant la mort?

À ma connaissance, les historiens québécois se sont peu penchés sur ces questions, autrement que pour signaler un trait pittoresque, rapporter une anecdote ou un fait divers. Ce sont les ethnologues et les folkloristes qui ont exploré le terrain. Déjà, il y a 25 ans, Madeleine Doyon fournissait une description détaillée des rites de la mort dans la Beauce (Journal of American Folklore, 67 (1954): 137-146). C'est l'une de ces pistes que vient d'explorer Paul Jacob, dans un mémoire de maîtrise qui a fort heureusement été publié, sur Les revenants de la Beauce. Les travaux se multiplient sur cette région dont la richesse traditionnelle et folklorique ne laisse pas d'étonner: qu'on pense aux publications récentes de Madeleine Ferron ou de Jean-Claude Dupont, pour n'en nommer que deux. Le volume de Jacob porte sur quelque 200 textes, recueillis dans huit paroisses entre Sainte-Marie et Beauceville (l'ancien Saint-François où Madeleine Doyon avait mené ses enquêtes). Un trait particulièrement intéressant du travail de Jacob, du point de vue pédagogique, est qu'il a su mettre à contribution les talents de recherche (ou de découverte) de jeunes de Secondaire V dans le cadre d'un cours. Le volume présente les principales constantes qui se dégagent des récits de revenants: comment se manifestent-ils? quand et où apparaissent-ils? pourquoi reviennent-ils sur terre? Il ressort de l'analyse que le revenant, la plupart du temps un parent ou voisin assez récemment décédé, se manifeste par des bruits (chaînes, mobilier, pas), sous forme d'apparition (homme sans tête, fantôme tout 
de blanc vêtu, ombre), ou de façon assez variée. Il apparaît surtout le soir, aux environs de la Toussaint ou quelque temps après son décès, le plus souvent dans la chambre de son «hôte». Le but de sa visite constitue le chapitre le plus intéressant. Il peut venir aider les vivants: ainsi une mère prêtera main-forte à ses enfants; plus fréquemment, le revenant viendra punir un survivant qui s'est moqué de lui. Mais le plus souvent, le revenant sollicite de l'aide: soit des prières ou des messes (déjà promises mais non réalisées), soit l'acquittement d'une dette ou le redressement d'un tort que le défunt n'avait pu régler de son vivant (un marché, un dérangement de bornes).

À cette bonne présentation, Paul Jacob a ajouté le texte intégral de 23 récits, merveilleusement transcrits, avec références précises sur la date de l'événement rapporté et les coordonnées de l'informateur. On regrette simplement que le texte ne renvoie pas au fur et à mesure aux récits, lorsque l'occasion se présente (ainsi p. 43). Ce qui frappe également dans ces récits, c'est la place de l'univers religieux. Ainsi, un survivant demande souvent à un mourant de lui fournir un signe (des «connaissances») après sa mort pour indiquer qu'il est «dans le bon chemin». On voit aussi des personnes demander (et obtenir) des faveurs à un défunt (une «âme du purgatoire») comme à un intercesseur aussi efficace qu'un saint. Enfin, il arrive fréquemment que ce soit le prêtre qui explique le sens de la manifestation d'un revenant, qu'on ne pouvait pas comprendre: le prêtre confirme, authentifie l'intervention qu'il qualifie de surnaturelle.

Voilà donc un volume qui éclaire très bien une facette, limitée mais significative, de la mort au Québec. L'historien regrettera que l'analyse soit menée sans qu'on tienne compte du facteur chronologique. Comme souvent dans les études folkloriques, on nous présente la réalité en un bloc, comme une matière éternelle ( $«$ traditionnelle» est le mot passe-partout qu'on emploie), sans se demander si la réalité étudiée a subi des variations dans le temps. Et c'est là, à mon avis, l'apport essentiel de la synthèse d'Ariès: les attitudes devant la mort varient, surtout il est vrai sur une longue durée. En terminant la Préface du livre de Jacob, Robert Cliche écrit: «Paul Jacob a fait un bon travail d'ethnologue. Il appartient aux psychologues et aux théologiens d'examiner la problématique de tout cela» (p. 11). Après avoir lu Ariès, nous serions porté à ajouter: et pourquoi pas aussi aux historiens? 